\title{
A Biomechanical Model of the Liver for Reality-Based Haptic Feedback ${ }^{1}$
}

\author{
Tie Hu and Jaydev P. Desai \\ Program for Robotics, Intelligent Sensing, and Mechatronics (PRISM) Laboratory \\ 3141 Chestnut Street, MEM Department, Room 2-115, Drexel University, Philadelphia, PA \\ 19104, USA \\ \{tie, desai\}@coe.drexel.edu
}

\begin{abstract}
Biomechanical model of soft tissue for remote probing based on observed experimental data is critical for developing a reality-based model for minimally invasive surgical training and simulation. In our research, we have focused on developing a biomechanical model of the liver with the ultimate goal of using this model for local tool-tissue interaction tasks and providing feedback through a haptic display. We have designed and developed tissue indentation equipment for characterizing the biomechanical properties of the liver and formulated a hybrid nonlinear model that is valid in both low strain and high strain regions. The pig liver is simplified as the incompressible, isotropic, and homogeneous elastic material. This model will be the basis for a finite element model for the pig liver.
\end{abstract}

\section{Introduction}

The goal of this paper is to develop a mathematical model for surface interactions with the pig liver based on observed experimental data. This model will be used for developing a reality-based haptic interface for tool-tissue interaction in MIS. "Global" elastic deformations of real and phantom tissues have been studied extensively in previous work, through simple poking interactions [1-3]. However, these methods are simplistic since they do not take into account the complex boundary conditions that are normally present, both internal to the organ and on the exterior surface. Howe and colleagues [4] have developed a "truth cube" for validation of models, but they have not studied tool-tissue interactions for common surgical tasks such as probing and cutting tissues. There has also been research on estimating the mechanical properties of the tissue through high-frequency shear deformations of the tissue sample, and elastography techniques. A variety of other techniques also exist in the literature for estimating the viscoelastic characterization of tissues, for example, [5-8].

For probing tasks, the quantitative knowledge of the biomechanical property of tissue is essential for soft tissue modeling. Fung [9] first showed that the elasticity property of rabbits' mesentery could be simply expressed as an exponential function. It

1 We would like to acknowledge the support of National Science Foundation grants: EIA0079830, and CAREER Award IIS-0133471 for this work. 
has been known that soft tissue has extremely nonlinear stress-strain relationship, which makes it difficult to model large strain deformations. To develop a liver model for the above tasks, it is necessary to study the biomechanical properties of the liver, which is valid for both small and large strain. In our research, we do not precondition the liver tissue prior to experimentation since surgeons interact with nonpreconditioned tissues and/or organs. As a first approach to model the liver tissue, we assume that the liver tissue is an incompressible (no volume change during deformation), homogeneous (same tissue composition throughout), and isotropic (no directional preference) elastic material. We have not included stress history in our modeling. Therefore, the stress of the liver tissue is only determined by the strain and the strain energy function can be obtained through the deformation tensor [10].

\section{Experimental Setup for Probing Liver Tissue}

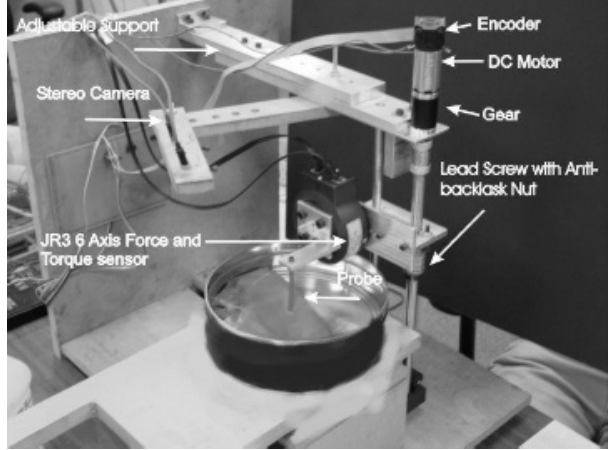

Fig. 1. Experiment setup for measuring force and displacement during probing

We have designed and developed a tissue probing apparatus, consisting of a motion control part, a force measuring part, and a post-data processing part to measure the probing force and displacement (see figure 1(a)). The motion control part is a lead screw assembled with a geared DC motor and encoder (Maxonmotor, Inc.), which is supported by two horizontal supports. The anti-backlash nut in the lead screw prevents any backlash in the mechanism. A precision JR3 6 axis force/torque sensor (model $85 \mathrm{M} 35 \mathrm{~A}-\mathrm{I} 40)$ is attached to the probe and it travels along the lead screw as shown in Figure 1. The position of the probe is controlled by the dSPACE DS1103 controller board (dSPACE, Inc.) and it also records the force and displacement data. The sampling frequency for control and data acquisition is $1000 \mathrm{~Hz}$. The algorithm implemented in the dSPACE card is a proportional +derivative (PD) control scheme. The communication between the dSPACE card and the JR3 data acquisition card is done by CLIB library (dSPACE, Inc.). We used probe head diameter of $2 \mathrm{~mm}$ for our tests. The livers were taken from freshly slaughtered pigs and transported to the lab within 2 hours post mortem. The liver was put in a container, which is filled with saline to ensure that the properties did not change dramatically over the next several hours. For the probing experiments, the liver was cut into specimen of size $50 \mathrm{~mm} \times 50 \mathrm{~mm}$ while the height was variable, as we did not want to cut through the liver capsule (preserving the capsule will provide us with realistic deformation forces for surface interactions with the liver). 


\section{Results from Probing Experiments}

In the probing experiment, we indented the liver with $2 \mathrm{~mm}$ probe diameter at varying probing speed. We used the small probe to observe localized deformations in the tis sue and develop our nonlinear model. We performed the probing experiment at two

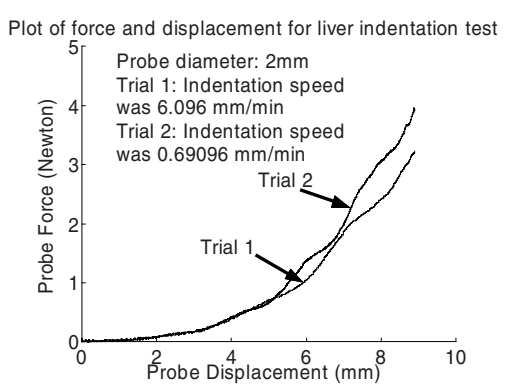

Fig. 2. Representative plot of force versus displacement for liver probing at two different speeds. speeds, namely, $6.096 \mathrm{~mm} / \mathrm{min}$ and 0.6096 $\mathrm{mm} / \mathrm{min}$ to see the effect of strain rate on the force versus displacement curve. From figure 2 we observe that the strain rate has little influence on the force versus displacement plot for a small probe. We did the probing experiment for a total of 4 trials. The tests were done on the same liver, but points at which probing was done were different (spaced far apart compared to the probe diameter to negate any effects from the other probing tests). Figure 2 shows force versus displacement plot for the small probe $(2 \mathrm{~mm})$. In all probing experiments, the tissue was deformed to $50 \%$ of

its nominal thickness. It is reasonable to carry out probing tests on the liver up to $50 \%$ of the nominal thickness at the point of probing since in surgery most tissue deformations on the organ surface are at most up to that range. Furthermore large deformation with the small probe can lead to tissue rupture, which is undesirable for the scope of this research. Analysis of the observed experimental data is presented in section 4 .

We did several experiments to de-

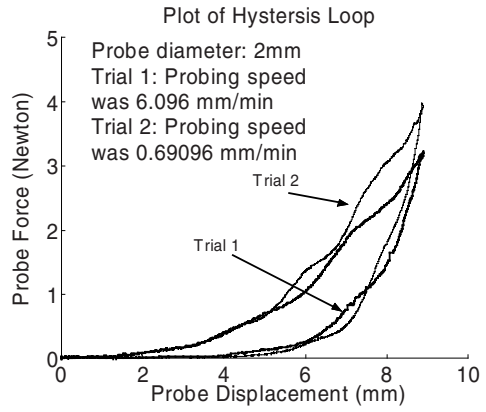

Fig. 3. Hysteresis plot at different strain rates. termine if the liver tissue was linear viscoelastic or not. Figure 3 shows force versus displacement for loading and unloading section. This was achieved by controlling the probe motion in the forward (probing) and reverse (probe moved away from the tissue surface) motion. The effect of the strain rate on the hysteresis plot is an important indication of the visoelasticity of the specimen. The probe size for the test was $2 \mathrm{~mm}$ diameter and the probing speeds in the two trials were $6.096 \mathrm{~mm} / \mathrm{min}$ and $0.6096 \mathrm{~mm} / \mathrm{min}$. The two trials used the same liver and the testing locations were at different points (spaced far apart compared to the probe diameter to negate any effects from the first hysteresis testing). From Figure 3, we observe that the hysteresis loop for varying strain rate has little effect on the force versus displacement curve. As a result we can conclude that the liver tissue is not liner viscoelastic. 


\section{Mathematical Model to Characterize Deformation Resistance to Probing}

This section describes a one-dimensional mathematical model for probing liver based on the experimental results presented in section 3.1. Figure 4 shows the schematic of the probing experiment. In this model, we will derive the stress versus strain relationship of liver tissue and show that it is nonlinear. We have assumed that the stress is only determined by the strain history. For a small probe, we can assume that the stress

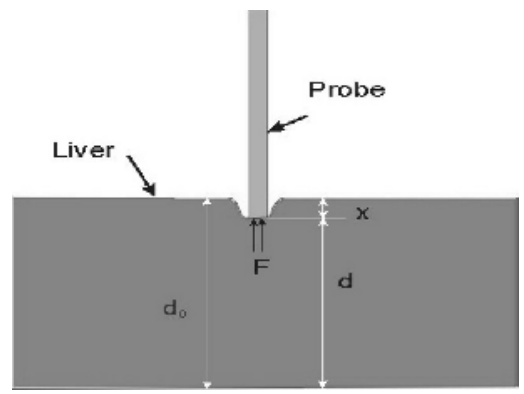

Fig. 4. Schematic of the indenting experiment.

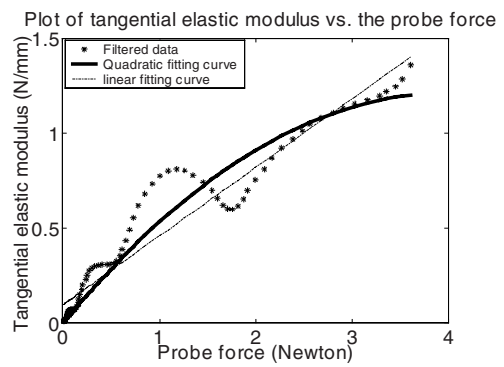

Fig. 5. Plot of tangential elastic modulus of the liver with probing force. Probe diameter was $4 \mathrm{~mm}$ and indentation speed was $0.6096 \mathrm{~mm} / \mathrm{min}$. under the probe can be considered as the median stress applied on the surface of the probe. Based on the above assumptions, the stress-strain relationship can be modeled as:

$$
\sigma(t)=F(\varepsilon(t))
$$

where $\sigma(t)$ is the stress on the specimen, $\varepsilon(t)$ is the corresponding strain history and $F(\bullet)$ is the function describing this nonlinear relationship.

The probing experiment results in Figure 2 show that the force versus displacement plot is nonlinear. Based on the probing results of figure 2 , we can take the derivative of the force with respect to the displacement to obtain the tangential elastic modulus of the specimen (with suitable scaling, since the strain is a function of $x$, as shown later in this section). The derivative data was filtered using a fifth order Butterworth filter with a cutoff frequency of $50 \mathrm{~Hz}$. Figure 5 shows the plot of the tangential elastic modulus of the specimen with respect to the probe force. The tangential elastic modulus of the specimen with respect to the probe force is almost linear at low forces, while at large force values, the plot is best fit by a quadratic curve. Based on this, we derived a hybrid mathematical model of load versus displacement for the

liver tissue specimen. This model has a linear and quadratic component. The linear component is valid for small strains (up to $16 \%$ compression) while the quadratic model is applicable for indentation up to $50 \%$ of the nominal thickness. This model was later validated with the observed experimental data of figure 2.

\subsection{Linear Model for Small Strain (Up to an Average 16\% Compression)}

A linear equation describing the probe force and the tangential elastic modulus can be modeled as: 


$$
\frac{\partial T}{\partial x}=a T+b
$$

where $T$ is the probe force, $x$ is the tissue displacement under the probe, and $a$ and $b$ are constants. Based on the initial conditions $T^{*}=T(0)=0$ and $x^{*}=x(0)=0$, and solving equation (2), we obtained the following relationship between the probe force and the corresponding displacement:

$$
T=\beta e^{\alpha x}+\gamma
$$

where $\alpha, \beta$, and $\gamma$ are model parameters expressed in terms of $a$ and $b$.

Based on the initial condition, Table 1 summarizes the calculated values of $\alpha, \beta$, and $\gamma$ for each trial and the average of four trials. Table 1 also presents the range of validity of the linear model, which was calculated based on determining the maximal ordinate $\bar{x}$, up to which the linear model was better than the quadratic model (presented below). Through post-processing of our experimental results, we found that the linear model was a better fit with the experimental data compared to the quadratic model for tissue compression up to $16 \%$ of the nominal thickness of the specimen.

Table 1. Linear and quadratic model parameters with the corresponding validity range for each model for four probing trials.

\begin{tabular}{|c|c|c|c|c|}
\hline \multicolumn{5}{|c|}{ Linear Model } \\
\hline Test \# & $\alpha$ & $\beta$ & $\gamma$ & Validity range \\
\hline 1 & 0.2715 & 0.3055 & -0.5231 & $0-13 \%$ \\
\hline 2 & 0.2993 & 0.1835 & -0.3033 & $0-17 \%$ \\
\hline 3 & 0.2161 & 0.4371 & -0.5452 & $0-20 \%$ \\
\hline 4 & 0.3670 & 0.1662 & -0.2907 & $0-15 \%$ \\
\hline Average & 0.2884 & 0.2730 & -0.4155 & $0-16 \%$ \\
\hline \multicolumn{5}{|c|}{ Quadratic Model } \\
\hline Test \# & $\mathrm{a}$ & $\mathrm{b}$ & $\mathrm{C}$ & Validity range \\
\hline 1 & 0.6583 & 0.1870 & 47.0144 & $13 \%-50 \%$ \\
\hline 2 & 0.6955 & 0.3157 & 78.4300 & $17 \%-50 \%$ \\
\hline 3 & 0.6045 & 0.2725 & 23.6000 & $20 \%-50 \%$ \\
\hline 4 & 0.6920 & 0.1680 & 44.2630 & $15 \%-50 \%$ \\
\hline Average & 0.6625 & 0.2358 & 48.3268 & $16 \%-50 \%$ \\
\hline
\end{tabular}

\subsection{Quadratic Model for Large Strain (Greater than 16\% Compression and up to $50 \%$ Compression)}

For large strain, we assumed the following nonlinear relationship between the elastic modulus and the probe force, $T$ : 


$$
\frac{\partial T}{\partial x}=c_{1} T^{2}+c_{2} T
$$

where $T$ and $x$ are described above and $c_{1}$ and $c_{2}$ are constants. We found the location of minimal error between the quadratic and linear model to determine the maximum ordinate $\bar{x}$. Thus the initial conditions for the quadratic model are: $T^{*}=T(\bar{x})$ and $x^{*}=\bar{x}$.

Solving the above differential equation for the quadratic model, we obtained the following relationship between the probe force and the corresponding displacement.

$$
T=\frac{e^{a x}}{C+b e^{a x}}
$$

where a, b, and $C$ are model parameters expressed in terms of $c_{1}$ and $c_{2}$. Table 1 summarizes the results of the calculated parameter values $a, b$, and $C$. As observed from the table, the quadratic model on the average was valid when the tissue was compressed from $16 \%$ to $50 \%$ of its nominal thickness.

\subsection{Hybrid Model (from $0 \%$ to $50 \%$ Compression)}

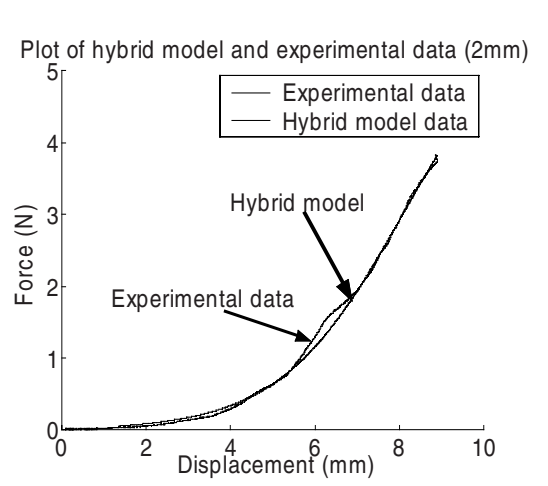

Fig. 6. Plot of hybrid model and experiment data.
Based on the linear and quadratic models proposed above, the hybrid model is given by:

$$
T(x)= \begin{cases}\beta e^{\alpha x}+\gamma & 0<x \leq \bar{x} \\ \frac{e^{a x}}{C+b e^{a x}} & -x \leq x<x_{0}\end{cases}
$$

where $x_{0}=\frac{d_{0}}{2}$ (based on $50 \%$ compression). Using equation (6), we plotted the theoretical load versus displacement curve and compared it with the experimental observation of figure 2. Figure 6 shows a representative plot of the theoretical and experimental load versus displacement curve. As seen from the figure, there is excellent match between the experimental and theoretical plot. Based on the validation of the above theoretical model, we can derive the stress vs. strain relationship for the liver specimen. The median stress at the probing point is given by:

$$
\sigma=\frac{T}{A_{0}}
$$

where $A_{0}$ is the probe area and $T$ is the compression load. For analysis, suppose there is a tissue cube of size $1 \times 1 \times 1$ units before compression and after subjecting the cube to one dimensional normal forces, the dimensions of the cube are $\lambda_{1} \times \lambda_{2} \times \lambda_{3}$ units. Since the liver is assumed to be an incompressible material, the 
volume of the cube will not change after this deformation. In our one-dimensional model, we will denote $\lambda=\lambda_{1}$ as the compression ratio.

Since $x$ is the probe displacement, $d$ is the depth of the deformed liver, and $d_{0}$ is the depth of the undeformed liver, the compression ratio $\lambda$ can be written as:

$$
\lambda=1-\frac{x}{d_{0}} \quad x(\sqrt{12 \varepsilon}) d_{0}
$$

From equations (6), (7), and (8), we can derive the hybrid stress versus strain relationship for pig liver to be:

$$
\sigma(\varepsilon)= \begin{cases}\frac{1}{A_{0}}\left(\beta e^{\alpha(1-\sqrt{1-2 \varepsilon}) d_{0}}+\gamma\right) & 0<\varepsilon \leq \varepsilon^{*} \\ \frac{1}{A_{0}}\left(\frac{e^{a(1-\sqrt{1-2 \varepsilon}) d_{0}}}{C+b e^{a(1-\sqrt{1-2 \varepsilon}) d_{0}}}\right) & \varepsilon^{*} \leq \varepsilon<\varepsilon^{0}\end{cases}
$$

where $\varepsilon^{*}$ is the maximum strain for the validity of the linear model, and $\varepsilon^{0}$ is the strain at $50 \%$ of nominal thickness. The strain energy of the liver can be obtained once the deformation of the tissue is known. The strain energy function, $U(x)$, is equal to the work done by the load, $T$ and is given by:

$$
U(x)=\frac{1}{A_{0} d_{0}} \int_{0}^{x} T(x) A_{0} d x
$$

where $A_{0}$ is the probe area, $T(x)$ is the compression load, $d_{0}$ is the depth of undeformed liver, and $x$ is the displacement of the probe. We get the following strain energy equation by substituting equation (6) into equation (10):

$$
U(x)= \begin{cases}\frac{\beta}{\alpha d_{0}} e^{\alpha x}+\frac{\gamma}{d_{0}} x-\frac{\beta}{\alpha d_{0}} & 0<x \leq \bar{x} \\ \frac{\ln \left(C+b e^{a x}\right)}{b a d_{0}}-\frac{\ln (C+b)}{b a d_{0}} & -x \leq x<x_{0}\end{cases}
$$

\section{Conclusions and Future Work}

We have designed and developed the indentation experiment setup for the pig liver, which can measure not only the interaction force, but also the deformation of the surrounding area of indentation. Like most soft tissues, liver tissue has an extremely nonlinear stress-strain relationship. It is thus critical to get the quantitative knowledge of the biomechanical properties of liver for a true haptic display of surface interactions with the liver. In this work, we assumed that the liver was an incompressible, isotropic, and homogeneous elastic material.

Based on the results from the indentation experiments, we developed a hybrid load vs. displacement relationship for the pig liver. The hybrid model matches very well with the experimental force vs. displacement data from low strain (up to $10 \%$ compression) to large strain (up to $50 \%$ compression). We also concluded that the liver is not linear viscoelastic since the load vs. displacement curve for loading and unloading was not dependent on the strain rate. Our experimental setup also had a pair 
of micro-cameras to capture the image of the dots on the liver surface during the deformation process. Though this data was not presented in this paper, we have captured this data for our future work on validating the FEM model that we are interested in developing. We are aware that in some cases, specular reflection and occlusion (by the probe) will prohibit us from obtaining the image of all the dots, we are nonetheless interested in obtaining the image of as many dots as possible since that information will be used later to validate the displacement of the surrounding nodes in the nonlinear FEM model.

The mathematical model proposed above will be useful for developing a realitybased nonlinear finite element liver organ model at the site of tool-tissue interaction.

\section{References}

1. d'Aulignac, D., R. Balaniuk, and C. Laugier, A Haptic Interface for a Virtual Exam of the Human Thigh. Proceedings of the IEEE International Conference on Robotics \& Automation, 2000: p. 2452-2456.

2. Kennedy, C.W., et al., A Novel approach to Robotic Cardiac Surgery using Haptics and Vision. Cardiovascular Engineering: An International Journal, 2002, 2002.

3. Brouwer, I., Measuring In Vivo Animal Soft Tissue Properties for Haptic Modeling in Surgical Simulation. Medicine Meets Virtual Reality, 2001: p. 69-74.

4. Kerdok, A.E., Soft Tissue Characterization: Mechanical Property Determination from Biopsies to Whole Organs. Whitaker Foundation Biomedical Research Conference, 2001.

5. Arbogast, K.B., et al., A High-Frequency shear device for testing soft biologicial tissues. Journal of Biomechanics, 1997. 30(7): p. 757-759.

6. Halperin, H.R., et al., Servo-Controlled Indenter for determining the transverse stiffness of ventricular muscle. IEEE Transactions on Biomedical Engineering, 1991. 38(6): p. 602-607.

7. Dokos, S., J.J. LeGrice, and B.H. Smaill, A Triaxial-Measurement Shear-Test Device for Soft Biological Tissues. Journal of Biomechanical Engineering, 2000. 122: p. 471-478.

8. LeGrice, I.J., Y. Takayama, and J.W. Covell, Transverse Shear Along Myocardial Cleavage Planes provides a mechanism for normal systolic wall thickening. Circulation Research, 1995. 77: p. 182-193.

9. Fung, Y.C., Biomechanics: Mechanical Properties of Living Tissues. 2nd ed. 1993, New York: Springer.

10. Atkin, R.J. and N. Fox, An Introduction to the theory of elasticity. 1980, London: Longman. 